\title{
陆地生态系统野外增温控制实验的技术与方法
}

\author{
朱 彪 ${ }^{*}$ 陈 迎
}

北京大学生态研究中心, 北京大学城市与环境学院, 北京大学地表过程分析与模拟教育部重点实验室, 北京 100871

摘 要 由于人类活动导致的碳排放急剧增加, 工业革命以来全球地表温度显著增加约 $1{ }^{\circ} \mathrm{C}$, 未来全球气候还将持续变暖, 到21世纪末最高可升温 $4{ }^{\circ} \mathrm{C}$ 。这种前所未有的气候变化不仅影响陆地植被的适应策略, 也深刻影响生态系统的结构和功能。 其中陆地生态系统碳收支对全球变暖的反馈, 是决定未来气候变化强度的关键因素, 因此全球已经开展了大量的生态系统尺 度的野外增温控制实验，研究生态系统碳收支对气温升高的响应，从而提高地球系统模型的预测精度。然而由于增温技术和 方法的不同, 不同研究的结果之间难以进行比较。该文系统总结了常见的野外增温技术和方法, 包括主动增温和被动增温, 阐述了其优缺点、适用对象以及相关研究成果。同时简要介绍了野外增温控制实验的前沿研究方向一新一代野外增温技术 (包括全土壤剖面增温和全生态系统增温)和基于新一代增温技术开展的野外增温联网实验。

关键词 陆地生态系统; 模拟增温; 增温技术; 碳收支; 深层土壤

朱彪, 陈迎 (2020). 陆地生态系统野外增温控制实验的技术与方法. 植物生态学报, 44, 330-339. DOI: 10.17521/cjpe.2019.0179

\section{Techniques and methods for field warming manipulation experiments in terrestrial ecosystems}

\section{ZHU Biao* and CHEN Ying}

Institute of Ecology, College of Urban and Environmental Sciences, and Key Laboratory for Earth Surface Processes of the Ministry of Education, Peking University, Beijing 100871, China

\begin{abstract}
Due to the sharp increase in carbon emissions from human activities, global surface air temperature has increased significantly by approximately $1{ }^{\circ} \mathrm{C}$ since the Industrial Revolution, and it will continue to increase by up to $4{ }^{\circ} \mathrm{C}$ by the end of 21st century. This unprecedented climate change will not only affect the adaptation strategies of terrestrial vegetation, but also profoundly affect the structure and function of terrestrial ecosystems. The feedbacks of terrestrial ecosystem carbon cycling to warming is the key factor controlling the speed of future climate change. Therefore, a large number of ecosystem-scale field warming manipulation experiments have been conducted globally to study the carbon budget of terrestrial ecosystems and to improve the prediction accuracy of earth system models. However, due to differences in techniques and methods of these field warming experiments, results among different studies are difficult to compare and synthesize. This paper reviews the common techniques and methods of field warming manipulation experiments, including active warming and passive warming. It also summarizes advantages and disadvantages, applicable objects and related publications for these techniques and methods. Moreover, it briefly introduces future directions of field warming manipulation experiments - the next-generation field warming techniques, namely whole-soil-profile warming and whole-ecosystem warming, and calls for establishing a coordinated distributed network of field warming manipulation experiments using these techniques.
\end{abstract}

Key words terrestrial ecosystems; simulated warming; warming techniques; carbon balance; deep soil

Zhu B, Chen Y (2020). Techniques and methods for field warming manipulation experiments in terrestrial ecosystems. Chinese Journal of Plant Ecology, 44, 330-339. DOI: 10.17521/cjpe.2019.0179

工业革命以来, 由于人为活动(包括化石燃料 燃烧和土地利用变化)导致的碳排放急剧增加, 全
球地表平均温度增加了约 $1{ }^{\circ} \mathrm{C}$ (IPCC, 2013)。而且, 根据政府间气候变化专门委员会(Intergovernmental

收稿日期Received: 2019-07-08 接受日期Accepted: 2019-09-16

基金项目：国家重点研发计划(2017YFC0503903)和国家自然科学基金(31622013和31971528)。Supported by the National Key R\&D Program of China (2017YFC0503903), and the National Natural Science Foundation of China (31622013 and 31971528 ).

* E-mail: biaozhu@pku.edu.cn 
Panel on Climate Change, IPCC)的预测, 到21世纪末 全球平均气温还将增加 $1.5-2.0{ }^{\circ} \mathrm{C}$, 最高可达 $4{ }^{\circ} \mathrm{C}$ (IPCC, 2013)。这种前所未有的气候变化不仅会影响 陆地植被的适应策略, 也将深刻影响生态系统的结 构和功能(牛书丽等, 2007)。作为全球碳循环的重要 组分, 陆地生态系统碳收支对控制大气 $\mathrm{CO}_{2}$ 浓度和 调节全球气候变化起着重要的作用。最近10年, 陆 地生态系统碳汇(净吸收)可以抵消所有人为活动碳 排放的30\%左右(Le Quere et al., 2018)。然而, 这部 分碳汇在未来气候变化的背景下能否持续, 是增加 还是降低甚至消失(变成碳源), 在地球系统模型的 预测中还存在极大的不确定性(Friedlingstein et al., 2014)。特别是, 陆地生态系统碳循环对气温升高的 反馈, 是决定未来气候变化强度的一个重要因素 (Heimann \& Reichstein, 2008; IPCC, 2013)。而提高 地球系统模型的预测精度, 迫切需要与生态系统尺 度的野外控制实验相结合, 特别是关键参数和过程 的校准和验证(Shaver et al., 2000; Bradford et al., 2016)。

全球变暖主要是由大气中温室气体反射地面长 波辐射导致的, 这种增强的辐射通过三种能量方式 (显热、潜热、土壤热通量)影响气候变化(牛书丽等, 2007)。运用不同增温方式(温室、开顶式同化箱、 红外辐射器、加热电缆)可以模拟全球变暖过程。因 此, 随着气候变化和陆地生态系统碳循环研究的兴 起(Ciais et al., 2013), 生态系统尺度的野外增温控 制实验在近30年得到了学术界的高度重视(牛书丽 等, 2007; Aronson \& McNulty, 2009)。其定义, 简单 来说, 就是在野外原位, 对一个完整的生态系统(包 括生产者植物、消费者动物、分解者土壤微生物和 其非生物环境)进行实验增温, 模拟生态系统的碳 循环(和其他过程)对增温的响应和反馈。实际操作 中, 由于条件和技术的限制, 往往只能对生态系统 的主要组分(比如植物和土壤)进行一定程度的增 温。根据增温能量来源的差别, 生态系统野外增温 实验技术可以分为两类, 被动增温技术和主动增温 技术。前者不需要电力, 通过各种方式(比如土壤移 位、温室或开顶箱)对生态系统进行增温。后者需要 电力, 通过红外辐射灯或土壤加热电缆主动释放热 量, 对生态系统进行增温。最近几年, 在传统的主动 增温技术(红外辐射灯或土壤加热电缆)的基础上, 发展了新一代的全土壤剖面增温技术和全生态系统
增温技术。然而不同的野外增温控制技术由于设计 和原理的差别, 其适用对象并不相同, 导致增温后 陆地生态系统各过程的响应并不一致。

因此，比较和总结不同野外增温装置的技术、 方法、优点、缺点、适用对象, 可以有效评估陆地 生态系统过程对于全球变暖的响应和适应。本文拟 对常见的野外增温装置进行总结, 包括主动增温和 被动增温, 指出其优缺点, 适用对象以及相关研究 成果。并介绍野外增温控制实验的前沿研究方向 一一新一代的野外增温技术(包括全土壤剖面增温 和全生态系统增温)和基于新一代增温技术开展的 野外增温联网实验。

\section{1 陆地生态系统野外增温实验的主要技术 及典型案例}

生态系统尺度的野外增温实验, 主要目的是研 究碳循环与气候变化之间的反馈关系, 兴起于 1990 年左右。1988年IPCC成立，1990年发布第一期评估 报告, 引起了政府和公众对气候变化和碳循环的极 大重视。学术界(主要是生态学家)也开始在全球不 同陆地生态系统(比如森林、草地、苔原、湿地等) 采用各种技术(比如温室、开顶箱、红外辐射灯、土 壤加热电缆等), 对生态系统进行原位增温, 针对碳 循环对气候变暖的响应和适应开展了深入系统的长 期研究(表1)。

\section{1 被动增温}

第一代的陆地生态系统野外增温实验技术是被 动增温, 主要适用于偏远的没有电力供应的生态系 统, 比如北极或者高山苔原。这种技术的主要优点 是操作简单、成本较低、可以有多个重复, 主要缺 点是样方较小、只适用于植物较矮的生态系统而且 只能对生态系统的部分进行增温。被动增温技术, 主要有三种, 即土壤移位、温室和开顶箱, 但也存在 其他的被动增温方式, 比如利用雪墙进行增温 (Plaza et al., 2019)。

\subsection{1 土壤移位增温}

土壤移位, 是指将一定体积的原状土壤(包括 地上植物), 从温度较低的地方(比如高海拔或高纬 度地区)移到温度较高的地方(比如低海拔或低纬度 地区)。由于两地气温(和土温)的差别, 可以模拟气 候变暖对生态系统碳循环过程的影响。需要指出的 是, 由于海拔梯度或纬度梯度上, 其他环境(比如降 
水、太阳辐射、大气 $\mathrm{CO}_{2}$ 分压)或生物要素(比如传粉 者、草食昆虫和动物)不完全一致, 因此测定的两地 生态系统过程之间的差异还受到温度之外的其他因 素的影响。

这种技术适用于植物较矮的生态系统, 比如苔 原和草地。具体操作是, 一般挖出完整的土块(包括 植物), 一部分放回原地(作为干扰对照), 一部分移 位到温度较高的地方(低海拔或低纬度地区, 模拟 增温), 或移位到温度较低的地方(高海拔或高纬度 地区, 模拟降温), 待其适应一段时间后, 监测生态 系统过程对增温或降温的响应。实际操作中, 需要 注意的细节是尽量保持原状土块(包括植物)的完整, 降低移位对包括土壤和植物在内的生态系统的干扰, 同时体积(包括样方面积和土壤深度)尽量大, 能够 代表该生态系统的植物和土壤等。

该技术虽然有一定局限, 但由于操作简单, 已
经有了不少应用。比如, Li等(2016)在青藏高原祁连 山北坡的高寒草甸, 采用原状土壤 $(100 \mathrm{~cm} \times$ $100 \mathrm{~cm} \times 40 \mathrm{~cm}$ )移位技术, 系统地研究了高寒草甸 碳氮循环过程对增温和降温的响应，发现植物不同 物候期(展叶期和开花期)对增温和降温的响应程度 不一致。Wu等(2012)在美国北亚利桑那州的荒漠化 草地, 采用原状土壤 $(30 \mathrm{~cm}$ 直径 $\times 30 \mathrm{~cm}$ 深度)移位 技术, 发现增温改变了植物物种组成, 加速了氮矿 化和氮流失, 并减弱了碳循环对气候变化的反馈。

\subsection{2 温室增温}

温室增温, 是采用简易温室(比如塑料薄膜覆 盖), 将地面释放的长波辐射部分反射回植物和表 层土壤, 从而对生态系统进行增温的技术。这与导 致全球气候变暖的温室效应原理相似, 因此能较好 地模拟气候变暖对生态系统的影响。但是, 由于温 室覆盖将生态系统与外面的环境进行了一定程度的

表1 陆地生态系统野外增温控制实验技术与方法总结

Table 1 Summary of techniques and methods for field warming manipulation experiments in terrestrial ecosystems

\begin{tabular}{|c|c|c|c|c|c|}
\hline $\begin{array}{l}\text { 技术 } \\
\text { Technique }\end{array}$ & $\begin{array}{l}\text { 方法 } \\
\text { Method }\end{array}$ & $\begin{array}{l}\text { 优点 } \\
\text { Advantage }\end{array}$ & $\begin{array}{l}\text { 缺点 } \\
\text { Disadvantage }\end{array}$ & $\begin{array}{l}\text { 适用对象 } \\
\text { Object }\end{array}$ & $\begin{array}{l}\text { 应用举例 } \\
\text { Example }\end{array}$ \\
\hline \multirow[t]{3}{*}{$\begin{array}{l}\text { 被动增温 } \\
\text { Passive } \\
\text { warming }\end{array}$} & $\begin{array}{l}\text { 土壤移位 } \\
\text { Soil translocation }\end{array}$ & $\begin{array}{l}\text { 成本低, 易操作, 方便多点多 } \\
\text { 重复 } \\
\text { Low cost, easy to operate, con- } \\
\text { venient for multiple locations } \\
\text { and replicates }\end{array}$ & $\begin{array}{l}\text { 小尺度, 有扰动, 不能用于 } \\
\text { 森林 } \\
\text { Small scale, disturbed, not } \\
\text { suitable for forests }\end{array}$ & $\begin{array}{l}\text { 植物和表层土壤增温, 任何生 } \\
\text { 态系统, 特别是草地 } \\
\text { Plants and surface soil warming, } \\
\text { any ecosystem, especially } \\
\text { grassland }\end{array}$ & $\begin{array}{l}\text { Wu et al., 2012; } \\
\text { Li et al., } 2016\end{array}$ \\
\hline & $\begin{array}{l}\text { 温室 } \\
\text { Greenhouse }\end{array}$ & $\begin{array}{l}\text { 成本低, 适用于偏远无电源 } \\
\text { 地区 } \\
\text { Low cost, suitable for remote } \\
\text { power-free areas }\end{array}$ & $\begin{array}{l}\text { 小尺度, 密闭系统, 不能用于 } \\
\text { 森林 } \\
\text { Small scale, closed system, not } \\
\text { suitable for forests }\end{array}$ & $\begin{array}{l}\text { 植物和表层土壤增温, 植物较 } \\
\text { 矮、没有电源的生态系统 } \\
\text { Plants and surface soil warming, } \\
\text { short-stature, power-free eco- } \\
\text { system }\end{array}$ & Sistla et al., 2013 \\
\hline & $\begin{array}{l}\text { 开顶式同化箱 } \\
\text { Open-top chamber }\end{array}$ & $\begin{array}{l}\text { 成本低, 多梯度增温, 适用于 } \\
\text { 偏远无电源地区 } \\
\text { Low cost, multi-gradient } \\
\text { warming, suitable for remote } \\
\text { power-free areas }\end{array}$ & $\begin{array}{l}\text { 小尺度, 半密闭系统, 不能用 } \\
\text { 于森林 } \\
\text { Small scale, closed system, not } \\
\text { suitable for forests }\end{array}$ & $\begin{array}{l}\text { 植物和表层土壤增温, 植物较 } \\
\text { 矮、没有电源的生态系统 } \\
\text { Plants and surface soil warming, } \\
\text { short-stature, power-free eco- } \\
\text { system }\end{array}$ & $\begin{array}{l}\text { Henry \& Molau, 1997; } \\
\text { Elmendorf et al., 2012; } \\
\text { Shi et al., } 2017\end{array}$ \\
\hline \multirow[t]{2}{*}{$\begin{array}{l}\text { 主动增温 } \\
\text { Active } \\
\text { warming }\end{array}$} & $\begin{array}{l}\text { 红外辐射器 } \\
\text { Infrared radiators }\end{array}$ & $\begin{array}{l}\text { 扰动少, 模拟真实情景 } \\
\text { Low disturbance, simulate real } \\
\text { warming scenario }\end{array}$ & $\begin{array}{l}\text { 成本较高, 小尺度, 不能加热 } \\
\text { 深层土壤, 样地面积较小 } \\
\text { High cost, small scale, cannot } \\
\text { heat deep soil, small plot area }\end{array}$ & $\begin{array}{l}\text { 植物和表层土壤增温, 植物较 } \\
\text { 矮、有电源的生态系统 } \\
\text { Plants and surface soil warming, } \\
\text { short-stature, power-accessible } \\
\text { ecosystem }\end{array}$ & $\begin{array}{l}\text { Harte et al., 1995; } \\
\text { Kimball et al., 2008, } \\
\text { 2018; Kimball \& } \\
\text { Conley, 2009; Wan et } \\
\text { al., 2009; Wang et al., } \\
\text { 2012; Harte et al., } \\
\text { 2015; Liu et al., } 2018\end{array}$ \\
\hline & $\begin{array}{l}\text { 加热电缆 } \\
\text { Heating cables }\end{array}$ & $\begin{array}{l}\text { 可以用于加热土壤, 特别是森 } \\
\text { 林土壤 } \\
\text { Can be used to heat soil, espe- } \\
\text { cially forest soil }\end{array}$ & $\begin{array}{l}\text { 成本较高, 小尺度, 不能加热 } \\
\text { 空气和深层土壤, 有一定扰动 } \\
\text { High cost, small scale, cannot } \\
\text { heat air and deep soil, some } \\
\text { disturbance }\end{array}$ & $\begin{array}{l}\text { 土壤增温, 有电源, 任何生态 } \\
\text { 系统, 特别是森林 } \\
\text { Soil warming, power-accessible, } \\
\text { any ecosystem, especially the } \\
\text { forest }\end{array}$ & $\begin{array}{l}\text { Melillo et al., 2011, } \\
\text { 2017; Lin et al., } 2018\end{array}$ \\
\hline \multirow[t]{2}{*}{$\begin{array}{l}\text { 新一代实验 } \\
\text { Next- } \\
\text { generation }\end{array}$} & $\begin{array}{l}\text { 全土壤剖面 } \\
\text { Whole-soil-profile }\end{array}$ & $\begin{array}{l}\text { 扰动少, 模拟真实土壤增温情 } \\
\text { 景, 包括深层土壤 } \\
\text { Low disturbance, simulate real } \\
\text { soil warming scenario, includ- } \\
\text { ing deep soil }\end{array}$ & $\begin{array}{l}\text { 成本较高, 尺度较小, 不能加 } \\
\text { 热空气 } \\
\text { High cost, smaller scale, no } \\
\text { heating of air }\end{array}$ & $\begin{array}{l}\text { 土壤增温, 有电源, 任何生态 } \\
\text { 系统 } \\
\text { Soil warming, power-accessible, } \\
\text { any ecosystem }\end{array}$ & $\begin{array}{l}\text { Hanson et al., 2011; } \\
\text { Hicks Pries et al., } 2017\end{array}$ \\
\hline & $\begin{array}{l}\text { 全生态系统 } \\
\text { Whole-ecosystem }\end{array}$ & $\begin{array}{l}\text { 扰动少, 最接近真实的生态系 } \\
\text { 统增温情景 } \\
\text { Low disturbance, closest to the } \\
\text { real ecosystem warming sce- } \\
\text { nario }\end{array}$ & $\begin{array}{l}\text { 成本很高, 尺度较小, 难推广, } \\
\text { 不能用于森林 } \\
\text { High cost, small scale, difficult } \\
\text { to use widely, not suitable for } \\
\text { forest }\end{array}$ & $\begin{array}{l}\text { 全生态系统增温, 有电源, 植 } \\
\text { 物较矮的生态系统 } \\
\text { Whole-ecosystem warming, } \\
\text { power-accessible, short-stature } \\
\text { ecosystem }\end{array}$ & $\begin{array}{l}\text { Wilson et al., 2016; } \\
\text { Gill et al., 2017; } \\
\text { Hanson et al., 2017; } \\
\text { Richardson et al., } 2018\end{array}$ \\
\hline
\end{tabular}


隔离, 会影响传粉者、草食者等与植物的交流和互 作, 而且对非生物环境(比如降水和光照)会有一定 的影响, 因此温室增温技术研究的是这些因素与温 度对生态系统过程的综合影响。

温室技术适用于偏远的无电力供应的植物较矮 的生态系统, 特别是苔原和草地。比如, 从1989年开 始在阿拉斯加的北极苔原生态系统, 采用温室 $(2.5 \mathrm{~m} \times 5.0 \mathrm{~m} \times 1.5 \mathrm{~m})$ 进行了长期的生态系统增温 实验。每年生长季开始时 (积雪融化后), 换上新的聚 乙烯薄膜, 开始增温; 生长季结束后, 将薄膜去掉, 非生长季不增温。最近的一项研究发现, 20年(19892008年)长期增温显著改变了北极苔原的植物群落 组成(维管植物特别是灌木增加, 苔藓和地衣减少), 但没有显著影响土壤碳储量(Sistla et al., 2013)。

\subsection{3 开顶箱增温}

开顶箱(open-top chamber, OTC)增温, 是采用 开顶式的各种材料(塑料、纤维板、玻璃等)和形状(六 边形、圆形等)的箱子, 将地面释放的长波辐射部分 反射回植物和表层土壤, 从而对生态系统进行增温 的技术。和温室相似, 开顶箱技术也对生态系统与 外面的环境进行了一定程度的隔离, 会影响温度之 外的其他环境(比如土壤水分)和生物(比如传粉者和 草食者)因素。并且由于材料、形状和高度等的差别, 不同开顶箱(包括内部不同位置)的增温效果有差别, 对其他因素的影响程度也不一样。这些间接影响, 在分析生态系统过程对增温的响应和反馈时, 应该 考虑在内。

开顶箱技术也适用于偏远的无电力供应的植物 较矮的生态系统, 特别是苔原和草地。比如, 国际苔 原实验网络(International Tundra Experiment, ITEX), 采用统一的方案, 基于开顶箱技术, 研究全球多个 苔原生态系统对增温(夏季气温增加 $1-3{ }^{\circ} \mathrm{C}$ )的响应 (Henry \& Molau, 1997)。研究发现, 增温对苔原植物 群落的影响取决于当地的气候条件和增温时间 (Elmendorf et al., 2012)。在温度较高的地点增温导 致灌木增加, 而在温度较低的地点增温导致禾草类 植物增加, 且这种变化随增温时间的延长没有饱和 的迹象。在中国青藏高原, 美国加州大学与中国科 学院西北高原生物研究所合作, 采用开顶箱技术开 展了国内第一个自然生态系统的野外增温实验。研究 发现, 16年(1997-2013年)增温(气温增加1.5 ${ }^{\circ} \mathrm{C}$ 左右, 表层10 cm土温增加 $1.0{ }^{\circ} \mathrm{C}$ 左右)之后, 高寒草甸和
高寒灌从生态系统的土壤碳储量并没有显著变化, 但是根系生物量分配向深层土壤转移, 有可能影响 深层土壤的碳氮水循环(余欣超等, 2015)。需要指出 的是, 通过控制开顶箱的特征(比如高度、直径、角 度等), 可以控制增温的强度, 研究不同增温幅度对 生态系统过程的影响(朱军涛, 2016; Shi et al., 2017)。

温室和开顶箱不仅可以用于增温处理, 而且还 可以应用于研究其他气候变化因子, 例如水分变 化、光照(强度和光质)和 $\mathrm{CO}_{2}$ 浓度变化等对生态系统 的影响(Hungate et al., 1997)。温室和开顶箱一般来 说可以增加空气温度 $1-3{ }^{\circ} \mathrm{C}$, 具体的温度要根据实 验目的和实际情况而定。如果利用电源加热, 补充 热空气进入开顶箱, 可以达到更均匀和更高的增温 效果(Norby et al., 1997)。

\section{2 主动增温}

第二代的生态系统野外增温实验技术是主动增 温, 主要是在有电力供应的生态系统, 采用悬挂在 植物上方的红外灯管或埋入土壤中的加热电缆, 对 生态系统进行原位增温。这种技术的主要优点是可 以控制能量输出和增温幅度, 扰动相对较小 (红外 灯管), 对土壤的增温效果较好(加热电缆), 主要缺 点是成本相对较高, 样地面积较小, 适用于植物较 矮的生态系统, 且只能对生态系统的部分进行增 温。包括两种主要技术, 分别为悬挂在植物上方的 红外灯管增温或埋入土壤中的加热电缆增温。

\subsection{1 红外辐射器增温}

红外辐射器增温, 是通过悬挂在样地上方, 可 以散发红外线辐射的灯管来对生态系统进行增温的 技术。该技术可以真实地模拟由于温室效应导致气 候变暖的机制, 即增强的向下红外线辐射。红外辐 射器的优点是从植物冠层上面加热, 能够在植被层 保持自然的温度梯度, 非破坏性地传递能量, 而且 不改变微环境。但是, 红外辐射器不直接加热空气, 不能模拟气候变暖的对流加热效应, 对于比较密集 的植被层可能会削弱对土壤的增温, 所能覆盖的面 积有限, 因此在森林生态系统中的应用受到限制 (牛书丽等, 2007)。

红外辐射器增温技术适用于有电力供应的植物 较矮的生态系统, 比如草地、农田和湿地。Harte等 (1995)在美国洛基山的亚高山草甸进行的增温实验 是最早采用这种技术(1990年开始)的实验, 在 $15 \mathrm{~W} \cdot \mathrm{m}^{-2}$ 的功率输入下, 悬挂在地面以上 $2.5 \mathrm{~m}$ 高度 
的红外辐射器 (160 cm长, $12 \mathrm{~cm}$ 宽), 最高可以增加 夏季土壤温度 $3.0{ }^{\circ} \mathrm{C}$, 降低土壤含水量 $30 \%$, 并导致 积雪融化期提前一周。基于该实验的结果表明, 增 温样地的土壤有机碳含量初期(5-6年)显著降低, 但 之后变化不大, 而对照样地的土壤有机碳含量在近 20年有持续下降趋势(Harte et al., 2015)。

红外辐射器增温技术自1990年以来, 在全球各 地的草地、农田和湿地等生态系统得到广泛的应用, 最近也被应用到森林生态系统的林下植物和土壤增 温(Kimball et al., 2018)。Kimball等(2015)对该技术 的原理做了深入分析, 并针对不同的生态系统做了 改进和调整(Kimball et al., 2008; Kimball \& Conley, 2009)。在我国，该技术也得到了广泛的应用。比如, 在北方温带草原, Wan等(2009)较早地采用该技术研 究了增温(和其他全球变化因子)对温带草地生态过 程的影响。在青藏高原也进行了类似的实验, 研究 增温对高寒草地生态过程的影响(Wang et al., 2012; Liu et al., 2018)。

需要指出的是, 红外辐射器增温技术, 如果采 用恒定功率输出, 则增温效果受到植被特征和气象 条件的影响(Harte et al., 1995); 如果结合实测温度 和反馈系统, 则可以精确控制增温的效果(Kimball et al., 2008)。具体采用哪种系统, 要视研究的具体 目标和预算而定。

\subsection{2 土壤加热电缆增温}

利用加热电缆可以对生态系统进行增温, 根据 研究目的和样地的不同, 加热电缆除了放置于土壤 上方, 还可置于土壤表层或者埋于土壤中(Aronson \& McNulty, 2009)。采用后者增温技术的实验居多, 因此此处主要介绍加热电缆运用到土壤中进行增温 的情形。土壤加热电缆增温, 是在土壤表层埋设加 热电缆, 通电之后对土壤进行主动增温的技术。这 种增温方式兴起于 20 世纪 90 年代, 得益于早期农业 和室内草坪中的土壤加热管道的启示。埋地电缆可 以通过土壤温度测定和电路程序控制得到一个稳定 可控的温度差, 它不像温室或开顶箱那样引起微气 候环境的改变。尽管这种装置需要电力, 在没有电 力设施的地方受到限制, 且对土壤有一定的扰动, 然而它是目前研究气候变暖影响森林生态系统(特 别是土壤生态过程)的可行手段(牛书丽等, 2007)。

这种技术主要应用在森林生态系统表层土壤增 温, 典型的实验是1991年开始的Harvard Forest土壤
增温实验。Peterjohn等(1993)采用埋设在10 cm深度 的加热电缆 $(6 \mathrm{~m}$ 长, 间距 $20 \mathrm{~cm}$, 样方 $6 \mathrm{~m} \times 6 \mathrm{~m}$, 重 复6个), 对土壤增温 $5{ }^{\circ} \mathrm{C}$, 研究了温带落叶阔叶林 的生态过程对气候变暖的响应。一项代表性的工作 是发现了土壤呼吸对增温的响应存在周期性的波动, 与土壤中碳底物质量和数量以及微生物群落组成和 生理活性的变化有关(Melillo et al., 2017)。另外, 为 了包括更多的树木, 测定更多生态系统尺度的碳氮 循环过程, Melillo等(2011)于 2001年开始建立了一 个 $30 \mathrm{~m} \times 30 \mathrm{~m}$ 的大样方 (由于资金限制, 只有 1 个重 复), 采用完全一样的设计, 研究了增温对土壤碳氮 循环和森林生产力的影响, 结果表明尽管7年土壤 增温会导致土壤呼吸增加, 但是由于植物碳库的增 加, 每年的生态系统净碳损失会随着增温年限的增 加而降低。在国内, 土壤加热电缆也被应用于森林 土壤的增温实验中。比如Lin等(2018)在亚热带人工 林和常绿阔叶林中进行的实验, 研究增温如何影响 不同土壤深度的 $\mathrm{CO}_{2}$ 排放, 发现由于深层土壤的贡 献导致增温后整个土壤剖面的碳排放增加 $40 \%$ 。

红外辐射器和土壤加热电缆两种增温技术也可 以结合使用, 可以对植物和土壤(表层)进行相对均 匀的增温。比如在美国明尼苏达, Rich等(2015)采用 地上悬挂红外灯管 $(1.6 \mathrm{~m}$ 高、8个等距的陶瓷加热器 与地面呈 $45^{\circ}$ 角)和地下 $10 \mathrm{~cm}$ 土壤埋设加热电缆, 对 林下植物和土壤进行了两个不同程度 $\left(1.7 、 3.4{ }^{\circ} \mathrm{C}\right)$ 的增温处理, 研究了气候变暖对温带-寒温带过渡 区的落叶阔叶林的林下幼苗光合作用和其他碳循环 过程的影响, 发现增温对树木光合作用的影响与土 壤水分状态有密切的关系(Reich et al., 2018)。

\section{2 研究前沿和热点}

在20多年的发展基础上, 最近几年生态系统尺 度的野外增温实验技术也出现了两种新一代的技术, 即对全部土壤剖面(0-1 m甚至0-3 m) 进行增温的全 土壤剖面增温技术(Hanson et al., 2011; Hicks Pries et al., 2017), 以及对包括地上空气、植物和地下全 部土壤剖面进行增温的全生态系统增温技术 (Hanson et al., 2017; Richardson et al., 2018)。这两种 新一代的生态系统尺度的增温技术, 如果在全球各 地同步开展有协调的联网实验(Fraser et al., 2013), 将极大地推动陆地生态系统碳循环与气候变化反馈 的研究(Torn et al., 2015)。 


\section{1 全土壤剖面增温}

由于深层土壤对生态系统碳循环的贡献越来越 受到重视(Rumpel \& Kögel-Knabner, 2011; Gross \& Harrison, 2019), 并且气候模型也预测深层土壤和 表层土壤的未来增温程度相似(Hicks Pries et al., 2018), 所以包括了深层土壤的全土壤剖面增温技 术近些年得到了广泛重视。全土壤剖面增温, 是在 圆形样方的四周, 垂直埋入多根(一般20-24根, 视 样方大小而定)加热电缆, 对全部土壤剖面进行均 匀增温的技术。该技术在2009年首次应用于温带落 叶阔叶林的增温预实验(Hanson et al., 2011), 1 个重 复, 样方直径是 $3.0 \mathrm{~m}$, 在 $3.5 \mathrm{~m}$ 直径(包括缓冲区 50 $\mathrm{cm})$ 的圆周, 均匀地将 24 根铁管和电缆垂直埋入地 下0-3 m, 通过测定不同深度的土壤温度和程序反 馈控制, 对整个土壤剖面 $(0-2 \mathrm{~m})$ 增温 $4{ }^{\circ} \mathrm{C}$ 。

应用该技术初步成功后, 2013年开始在美国加 利福尼亚州针叶林生态系统进行了有重复的全土壤 剖面增温实验(Hicks Pries et al., 2017), 对原技术的 细节做了微调, 比如改为 22 根铁管和电缆, 垂直埋 入地下 $0-2.4 \mathrm{~m}$ 深度, 同时为弥补表层土壤的热损 失(因为空气没有增温), 在 $1 \mathrm{~m}$ 和 $2 \mathrm{~m}$ 直径的样方四 周, $5 \mathrm{~cm}$ 土壤深度, 水平埋入两圈加热电缆。结果显 示, 对10-100 cm的土壤可以均匀地增温 $4{ }^{\circ} \mathrm{C}$, 表层 0-10 cm的增温效果较弱 $\left(2-3{ }^{\circ} \mathrm{C}\right)$ 。基于该技术, 他 们发现该针叶林不同深度的土壤呼吸对增温的响应 没有显著差别, 并且相比传统的只对表层土壤增温 的实验结果, 这种包括了深层土壤的全土壤剖面增 温处理会引起更多的土壤碳排放(Hicks Pries et al., 2017)。

目前，国际上已有5-6个团队在全球不同的生 态系统, 采用该技术研究土壤碳循环(特别是深层 土壤)对增温的响应和反馈, 并联合发起了国际土 壤实验网络(Torn et al., 2015)。国内也有一些团队开 始这方面的研究, 比如北京大学采用该技术, 研究 青海海北站的高寒草甸生态系统碳循环对全土壤剖 面(0-100 cm)增温 $4{ }^{\circ} \mathrm{C}$ 的响应(图1)。

当预算受限的时候, 可以对该技术做些改变, 对较小体积的土壤进行全剖面增温。比如对加州草 地生态系统, 采用lysimeter的设计, 通过在中心位 置垂直埋入一根加热电缆, 可以对野外的完整土柱 (38 cm直径、 $42 \mathrm{~cm}$ 深)相对均匀地增温 $4{ }^{\circ} \mathrm{C}$, 研究土 壤碳循环(包括 $\mathrm{CO}_{2}$ 释放和可溶性有机碳(DOC)淋溶)
对增温的响应(Castanha et al., 2018)。

\section{2 全生态系统增温}

全生态系统增温, 是对包括地上植物和地下全 部土壤在内的全生态系统进行增温的技术。由于早 期的被动或主动增温技术均有各种不足(表1), 不能 对整个生态系统进行全组分增温, 所以美国能源部 资助 Paul Hanson领导的团队, 在早期预实验 (Hanson et al., 2011)的基础上，自2015年开始在明 尼苏达州的云杉(Picea mariana)林-泥炭地生态系统, 开展了包括 5 个温度水平以及 2 个 $\mathrm{CO}_{2}$ 浓度的野外大 型实验(Spruce and Peatland Responses Under Changing Environments, SPRUCE)(Hanson et al., 2017)。

SPURCE实验的样方直径为 $12 \mathrm{~m}$, 采用 $8 \mathrm{~m}$ 高 度的开顶箱和热空气对地上植物和空气增温, 并采 用3圈(半径分别为 $5.42 、 4.00 、 2.00 \mathrm{~m}$ ) 垂直埋入 $0-3 \mathrm{~m}$ 土壤(泥炭)的电缆(分别是48、12、6根)对地下全土 壤剖面进行增温。根据不同深度土壤温度的测定数 据和计算机程序反馈控制电缆和热空气，可以对整 个生态系统进行不同程度(对照, 增温2.25、4.50、 6.75、9.00 ${ }^{\circ} \mathrm{C}$ )的增温处理(图2)。2015年开始运行以 来, 取得了较为良好的效果(Hanson et al., 2017)。比 如, 全生态系统增温导致木本植物的春季展叶期提 前, 秋季落叶期推迟, 且这种变化随增温幅度呈现 线性趋势, 表明光周期对该生态系统木本植物的物 候期没有显著影响(Richardson et al., 2018); 深层泥 炭地增温会导致 $\mathrm{CH}_{4}$ 和 $\mathrm{CO}_{2}$ 排放显著增加, 而且 $\mathrm{CH}_{4}$ 排放对温度增加更加敏感(Wilson et al., 2016; Gill et al., 2017)。

全生态系统增温技术, 相对来说成本较高, 操 作起来较复杂, 但是包括生态系统全部组分, 最接 近真实增温情景, 因此是最先进、最前沿的生态系 统尺度的野外增温实验技术。利用全生态系统增温 技术对生态系统进行增温实验, 能够更加准确地了 解陆地生态系统碳循环过程对增温的响应和适应, 因此建议国家科研管理部门对该技术重点资助, 特 别是在碳储量较高, 对气候变化比较敏感, 研究基 础较薄弱的生态系统, 比如高寒草地、泥炭地或湿 地等。

\section{3 野外增温联网实验}

虽然目前已经开展了大量的野外增温控制实验, 但是由于不同的增温实验具有不同的增温方式，而 且增温方式可能会影响陆地生态系统过程对增温的 

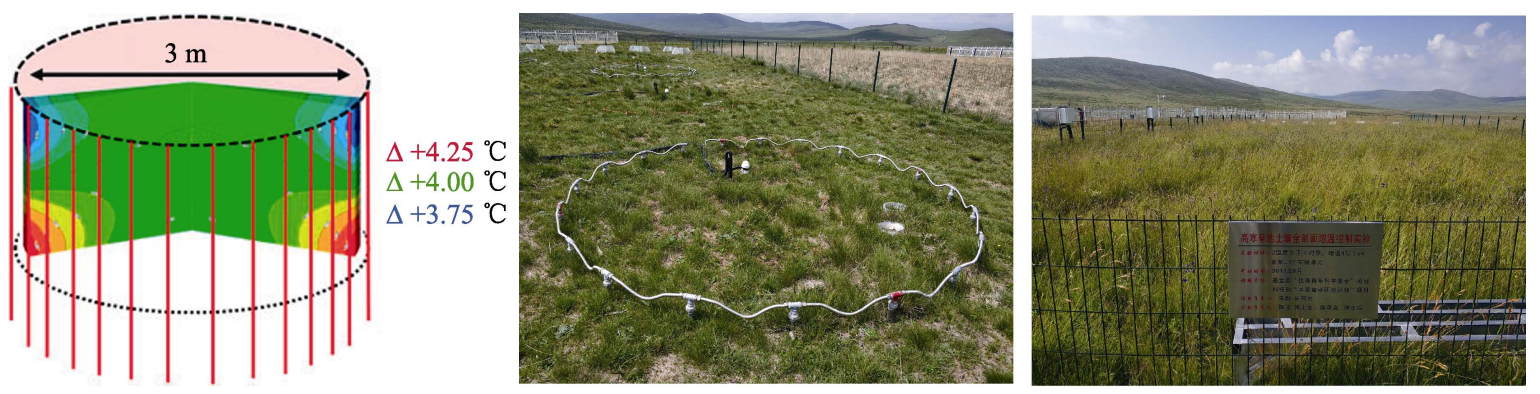

图1 青藏高原高寒草甸(海北)土壤全剖面增温实验。

Fig. 1 Whole-soil-profile warming experiment in the alpine meadow ecosystem (Haibei) on the Qinghai-Xizang Plateau, China.
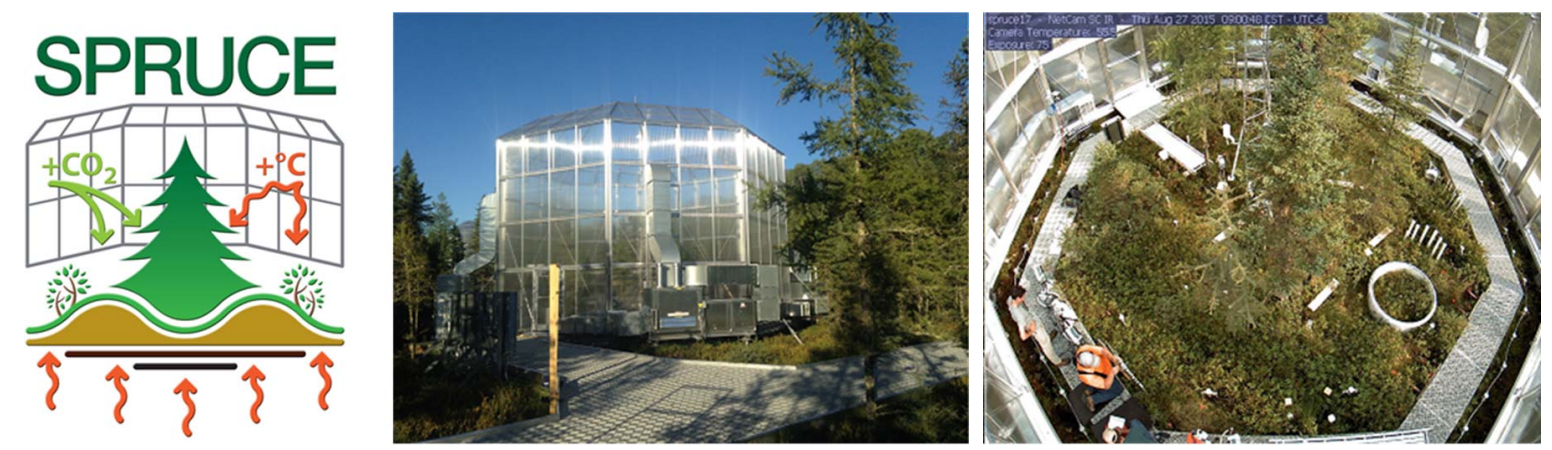

图2 美国明尼苏达州泥炭森林全生态系统增温实验(Hanson et al., 2017)。美国能源部资助的SPRUCE项目(美国能源部Oak ridge国家实验室, https://mnspruce.ornl.gov/)。

Fig. 2 Whole-ecosystem warming experiment in the peatland forest, Minnesota, USA (Hanson et al., 2017). The SPRUCE project was funded by the Department of Energy of USA (Courtesy of Oak Ridge National Laboratory, U.S. Dept. of Energy, https://mnspruce.ornl.gov/).

响应(Lu et al., 2013; Chen et al., 2015), 因此不同野 外增温控制实验的研究结果之间并不可比。同时由 于生态系统的空间异质性, 单个地点、单个生态系 统的控制实验结果在外推或验证模型时, 有较大的 不确定性, 因此迫切需要在多个地点、多个生态系 统, 采用统一的有协调的方法和技术, 进行联网实 验(Luo et al., 2011; Fraser et al., 2013)。最近10年, 由 于方法简单成本较低, 国际上已经针对降水变化和 养分变化开展了全球联网实验, 并且取得了一大批 高水平的研究成果(Knapp et al., 2015; Harpole et al., 2016)。因此, 未来的一个重要前沿, 是采用统一的 技术, 特别是新一代的全土壤剖面增温和全生态系 统增温技术, 开展全国甚至全球尺度的联网实验 (Torn et al., 2015)。这些实验, 将极大地推动陆地生 态系统碳循环响应气候变化的研究, 为深入了解生 态过程(特别是深层土壤过程、植物-土壤-微生物互 作过程)、显著提高碳循环模型的预测可靠性提供实 测数据。

\section{3 总结和展望}

生态系统尺度的野外增温控制实验已经有30年
左右的积累, 从早期的被动增温技术发展到主动增 温技术, 再到最近几年兴起的全土壤剖面增温和全 生态系统增温技术。这些技术已被应用在全球多个 生态系统, 显著促进了陆地生态系统碳循环对气候 变化响应和反馈的研究。然而, 现有的实验研究还存 在不足, 比如样点分布不均, 对土壤碳密度较大的 苔原、湿地和泥炭地以及植被碳储量较高的热带雨 林的研究尤其偏少, 在北美、欧洲和东亚之外的其他 地区也很少; 长期模拟增温实验较少, 特别是超过 10年的长期实验; 采用统一技术包括多个地点的联 网实验也相对不足。同时, 野外增温控制实验本身也 存在缺陷, 即其带来的瞬间增温不能很好地反映全 球未来的缓慢增温过程。因此, 今后的研究除了继续 采用已有的被动和主动增温技术, 维持已有的长期 实验, 还应该在研究薄弱的生态系统和地点(比如高 寒草地和苔原、泥炭地和湿地、亚热带常绿阔叶林 和热带雨林等), 采用新一代的技术手段(特别是全土 壤剖面增温和全生态系统增温技术), 针对以往研究 相对薄弱的生态学过程(比如深层土壤碳循环、植物土壤-微生物互作等)进行长期联网实验, 从而更好地 研究陆地生态系统碳循环等生态过程对气候变暖的

www.plant-ecology.com 
响应和反馈。这些新一代的生态系统尺度野外增温 联网控制实验, 将极大推动陆地生态系统碳循环对 气候变化响应和反馈的研究。

\section{参考文献}

Aronson EL, McNulty SG (2009). Appropriate experimental ecosystem warming methods by ecosystem, objective, and practicality. Agricultural and Forest Meteorology, 149, 1791-1799.

Bradford MA, Wieder WR, Bonan GB, Fierer N, Raymond PA, Crowther TW (2016). Managing uncertainty in soil carbon feedbacks to climate change. Nature Climate Change, 6, 751-758.

Castanha C, Zhu B, Hicks Pries CE, Georgiou K, Torn MS (2018). The effects of heating, rhizosphere, and depth on root litter decomposition are mediated by soil moisture. Biogeochemistry, 137, 267-279.

Chen J, Luo YQ, Xia JY, Jiang LF, Zhou XH, Lu M, Liang JY, Shi Z, Shelton S, Cao JJ (2015). Stronger warming effects on microbial abundances in colder regions. Scientific Reports, 5, 18032. DOI: 10.1038/srep18032.

Ciais P, Sabine C, Bala G, Bopp L, Brovkin V, Canadell J, Chhabra A, DeFries R, Galloway J, Heimann M, Jones C, Le Quéré C, Myneni RB, Piao SL, Thornton P (2013). Carbon and other biogeochemical cycles//Contribution of Working Group I to the Fifth Assessment Report of the Intergovernmental Panel on Climate Change. Climate Change 2013: the Physical Science Basis. Cambridge University Press, Cambridge, UK.

Elmendorf SC, Henry GHR, Hollister RD, Björk RG, Bjorkman AD, Callaghan TV, Collier LS, Cooper EJ, Cornelissen JHC, Day TA, Fosaa AM, Gould WA, Grétarsdóttir J, Harte J, Hermanutz L, Hik DS, Hofgaard A, Jarrad F, Jónsdóttir IS, Keuper F, Klanderud K, Klein JA, Koh S, Kudo G, Lang SI, Loewen V, May JL, Mercado J, Michelsen A, Molau U, Myers-Smith IH, Oberbauer SF, Pieper S, Post E, Rixen C, Robinson CH, Schmidt NM, Shaver GR, Stenström A, Tolvanen A, Totland Ø, Troxler $\mathrm{T}$, Wahren $\mathrm{CH}$, Webber PJ, Welker JM, Wookey PA (2012). Global assessment of experimental climate warming on tundra vegetation: heterogeneity over space and time. Ecology Letters, 15, 164-175.

Fraser LH, Henry HA, Carlyle CN, White SR, Beierkuhnlein C, Cahill Jr JF, Casper BB, Cleland E, Collins SL, Dukes JS, Knapp AK, Lind E, Long RJ, Luo YQ, Reich PB, Smith MD, Sternberg M, Turkington R (2013). Coordinated distributed experiments: an emerging tool for testing global hypotheses in ecology and environmental science. Frontiers in Ecology and the Environment, 11, 147-155.

Friedlingstein P, Meinshausen M, Arora VK, Jones CD, Anav A, Liddicoat SK, Knutti R (2014). Uncertainties in CMIP5 climate projections due to carbon cycle feedbacks. Journal of Climate, 27, 511-526.

Gill AL, Giasson MA, Yu R, Finzi AC (2017). Deep peat warming increases surface methane and carbon dioxide emissions in a black spruce-dominated ombrotrophic bog. Global Change Biology, 23, 5398-5411.

Gross CD, Harrison RB (2019). The case for digging deeper: Soil organic carbon storage, dynamics, and controls in our changing world. Soil Systems, 3, 28. DOI: 10.3390/ soilsystems3020028.

Hanson PJ, Childs KW, Wullschleger SD, Riggs JS, Thomas WK, Todd DE, Warren JM (2011). A method for experimental heating of intact soil profiles for application to climate change experiments. Global Change Biology, 17, 1083-1096.

Hanson PJ, Riggs JS, Nettles WR, Phillips JR, Krassovski MB, Hook LA, Gu LH, Richardson AD, Aubrecht DM, Ricciuto DM, Warren JM, Barbier C (2017). Attaining whole-ecosystem warming using air and deep-soil heating methods with an elevated $\mathrm{CO}_{2}$ atmosphere. Biogeosciences, 14, 861-883.

Harpole WS, Sullivan LL, Lind EM, Firn J, Adler PB, Borer ET, Chase J, Fay PA, Hautier Y, Hillebrand H, MacDougall AS, Seabloom EW, Williams R, Bakker JD, Cadotte MW, Chaneton EJ, Chu CJ, Cleland EE, D’Antonio C, Davies KF, Gruner DS, Hagenah N, Kirkman K, Knops JMH, La Pierre KJ, McCulley RL, Moore JL, Morgan JW, Prober SM, Risch AC, Schuetz M, Stevens CJ, Wragg PD (2016). Addition of multiple limiting resources reduces grassland diversity. Nature, 537, 93-96.

Harte J, Saleska SR, Levy C (2015). Convergent ecosystem responses to 23-year ambient and manipulated warming link advancing snowmelt and shrub encroachment to transient and long-term climate-soil carbon feedback. Global Change Biology, 21, 2349-2356.

Harte J, Torn MS, Chang FR, Feifarek B, Kinzig AP, Shaw R, Shen K (1995). Global warming and soil microclimate: results from a meadow-warming experiment. Ecological Applications, 5, 132-150.

Heimann M, Reichstein M (2008). Terrestrial ecosystem carbon dynamics and climate feedbacks. Nature, 451, 289-292.

Henry GHR, Molau U (1997). Tundra plants and climate change: the International Tundra Experiment (ITEX). Global Change Biology, 3(S1), 1-9.

Hicks Pries CE, Castanha C, Porras R, Phillips C, Torn MS (2018). Response to Comment on "The whole-soil carbon flux in response to warming”. Science, 359, eaao0457. DOI: $10.1126 /$ science.aao0457.

Hicks Pries CE, Castanha C, Porras RC, Torn MS (2017). The whole-soil carbon flux in response to warming. Science, 355, 1420-1422.

Hungate BA, Holland EA, Jackson RB, Chapin III FS, Mooney 
HA, Field CB (1997). The fate of carbon in grasslands under carbon dioxide enrichment. Nature, 388, 576-579.

IPCC (2013). Climate Change: the Physical Science Basis. Cambridge University Press, Cambridge, UK.

Kimball BA, Alonso-Rodríguez AM, Cavaleri MA, Reed SC, González G, Wood TE (2018). Infrared heater system for warming tropical forest understory plants and soils. Ecology and Evolution, 8, 1932-1944.

Kimball BA, Conley MM (2009). Infrared heater arrays for warming field plots scaled up to 5-m diameter. Agricultural and Forest Meteorology, 149, 721-724.

Kimball BA, Conley MM, Wang SP, Lin XW, Luo CY, Morgan J, Smith D (2008). Infrared heater arrays for warming ecosystem field plots. Global Change Biology, 14, 309-320.

Kimball BA, White JW, Ottman MJ, Wall GW, Bernacchi CJ, Morgan J, Smith DP (2015). Predicting canopy temperatures and infrared heater energy requirements for warming field plots. Agronomy Journal, 107, 129-141.

Knapp AK, Hoover DL, Wilcox KR, Avolio ML, Koerner SE, La Pierre KJ, Loik ME, Luo YQ, Sala OE, Smith MD (2015). Characterizing differences in precipitation regimes of extreme wet and dry years: implications for climate change experiments. Global Change Biology, 21, 2624-2633.

Le Quéré C, Andrew RM, Friedlingstein P, Sitch S, Pongratz J, Manning AC, Korsbakken JI, Peters GP, Canadell JG, Jackson RB, Boden TA, Tans PP, Andrews OD, Arora VK, Bakker DCE, Barbero L, Becker M, Betts RA, Bopp L, Chevallier F, Chini LP, Ciais P, Cosca CE, Cross J, Currie K, Gasser T, Harris I, Hauck J, Haverd V, Houghton RA, Hunt CW, Hurtt G, Ilyina T, Jain AK, Kato E, Kautz M, Keeling RF, Klein Goldewijk K, Körtzinger A, Landschützer P, Lefèvre N, Lenton A, Lienert S, Lima I, Lombardozzi D, Metzl N, Millero F, Monteiro PMS, Munro DR, Nabel JEMS, Nakaoka SI, Nojiri Y, Padin XA, Peregon A, Pfeil B, Pierrot D, Poulter B, Rehder G, Reimer J, Rödenbeck C, Schwinger J, Séférian R, Skjelvan I, Stocker BD, Tian H, Tilbrook B, Tubiello FN, van der Laan-Luijkx IT, van der Werf GR, van Heuven S, Viovy N, Vuichard N, Walker AP, Watson AJ, Wiltshire AJ, Zaehle S, Zhu D (2018). Global carbon budget 2017. Earth System Science Data, 10, 405-448.

Li XN, Jiang LL, Meng FD, Wang SP, Niu HS, Iler AM, Duan JC, Zhang ZH, Luo CY, Cui SJ, Zhang LR, Li YM, Wang Q, Zhou Y, Bao XY, Dorji T, Li YN, Peñuelas J, Du MY, Zhao XQ, Zhao L, Wang GJ (2016). Responses of sequential and hierarchical phenological events to warming and cooling in alpine meadows. Nature Communications, 7, 12489. DOI: 10.1038/ncomms12489.

Lin WS, Li YQ, Yang ZJ, Giardina CP, Xie JS, Chen SD, Lin CF, Kuzyakov Y, Yang YS (2018). Warming exerts greater impacts on subsoil than topsoil $\mathrm{CO}_{2}$ efflux in a subtropical forest. Agricultural and Forest Meteorology, 263, 137-146.

Liu HY, Mi ZR, Lin L, Wang YH, Zhang ZH, Zhang FW, Wang H, Liu LL, Zhu B, Cao GM, Zhao XQ, Sanders NJ, Classen AT, Reich PB, He JS (2018). Shifting plant species composition in response to climate change stabilizes grassland primary production. Proceedings of the National Academy of Sciences of the United States of America, 115, 4051-4056.

Lu M, Zhou XH, Yang Q, Li H, Luo YQ, Fang CM, Chen JK, Yang X, Li B (2013). Responses of ecosystem carbon cycle to experimental warming: a meta-analysis. Ecology, 94, 726-738.

Luo YQ, Melillo J, Niu SL, Beier C, Clark JS, Classen AT, Davidson E, Dukes JS, Evans RD, Field CB, Czimczik CI, Keller M, Kimball BA, Kueppers LM, Norby RJ, Pelini SL, Pendall E, Rastetter E, Six J, Smith M, Tjoelker MG, Torn MS (2011). Coordinated approaches to quantify long-term ecosystem dynamics in response to global change. Global Change Biology, 17, 843-854.

Melillo JM, Butler S, Johnson J, Mohan J, Steudler P, Lux H, Burrows E, Bowles F, Smith R, Scott L, Vario C, Hill T, Burton A, Zhou YM, Tang J (2011). Soil warming, carbon-nitrogen interactions, and forest carbon budgets. Proceedings of the National Academy of Sciences of the United States of America, 108, 9508-9512.

Melillo JM, Frey SD, DeAngelis KM, Werner WJ, Bernard MJ, Bowles FP, Pold G, Knorr MA, Grandy AS (2017). Long-term pattern and magnitude of soil carbon feedback to the climate system in a warming world. Science, 358, 101-105.

Niu SL, Han XG, Ma KP, Wan SQ (2007). Field facilities in global warming and terrestrial ecosystem research. Journal of Plant Ecology (Chinese Version), 31, 262-271. [牛书 丽, 韩兴国, 马克平, 万师强 (2007). 全球变暖与陆地 生态系统研究中的野外增温装置. 植物生态学报, 31, 262-271.]

Norby R, Edwards N, Riggs J, Abner C, Wullschleger S, Gunderson C (1997). Temperature-controlled open-top chambers for global change research. Global Change Biology, 3, 259-267.

Peterjohn WT, Melillo JM, Bowles FP, Steudler PA (1993). Soil warming and trace gas fluxes: experimental design and preliminary flux results. Oecologia, 93, 18-24.

Plaza C, Pegoraro E, Bracho R, Celis G, Crummer KG, Hutchings JA, Hicks Pries CE, Mauritz M, Natali SM, Salmon VG, Schädel C, Webb EE, Schuur EAG (2019). Direct observation of permafrost degradation and rapid soil carbon loss in tundra. Nature Geoscience, 12, 627-631.

Reich PB, Sendall KM, Stefanski A, Rich RL, Hobbie SE,

www.plant-ecology.com 
Montgomery RA (2018). Effects of climate warming on photosynthesis in boreal tree species depend on soil moisture. Nature, 562, 263-267.

Rich RL, Stefanski A, Montgomery RA, Hobbie SE, Kimball BA, Reich PB (2015). Design and performance of combined infrared canopy and belowground warming in the B4WarmED (Boreal Forest Warming at an Ecotone in Danger) experiment. Global Change Biology, 21, 2334-2348.

Richardson AD, Hufkens K, Milliman T, Aubrecht DM, Furze ME, Seyednasrollah B, Krassovski MB, Latimer JM, Nettles WR, Heiderman RR, Warren JM, Hanson PJ (2018). Ecosystem warming extends vegetation activity but heightens vulnerability to cold temperatures. Nature, 560, 368-371.

Rumpel C, Kögel-Knabner I (2011). Deep soil organic matter-A key but poorly understood component of terrestrial C cycle. Plant and Soil, 338, 143-158.

Shaver GR, Canadell J, Chapin FS, Gurevitch J, Harte J, Henry G, Ineson P, Jonasson S, Melillo J, Pitelka L, Rustad L (2000). Global warming and terrestrial ecosystems: a conceptual framework for analysis. BioScience, 50, 871-882.

Shi GX, Yao BQ, Liu YJ, Jiang SJ, Wang WY, Pan JB, Zhao XQ, Feng HY, Zhou HK (2017). The phylogenetic structure of AMF communities shifts in response to gradient warming with and without winter grazing on the QinghaiTibet Plateau. Applied Soil Ecology, 121, 31-40.

Sistla SA, Moore JC, Simpson RT, Gough L, Shaver GR, Schimel JP (2013). Long-term warming restructures Arctic tundra without changing net soil carbon storage. Nature, 497, 615-618.

Torn MS, Chabbi A, Crill P, Hanson PJ, Janssens IA, Luo Y, Hicks Pries CE, Rumpel C, Schmidt MWI, Six J, Schrumpf M, Zhu B (2015). A call for international soil experiment networks for studying, predicting, and manag- ing global change impacts. Soil, 1, 575-582.

Wan SQ, Xia JY, Liu WX, Niu SL (2009). Photosynthetic overcompensation under nocturnal warming enhances grassland carbon sequestration. Ecology, 90, 2700-2710.

Wang SP, Duan JC, Xu GP, Wang YF, Zhang ZH, Rui YC, Luo CY, Xu B, Zhu XX, Chang XF, Cui XY, Niu HS, Zhao XQ, Wang WY (2012). Effects of warming and grazing on soil $\mathrm{N}$ availability, species composition, and ANPP in an alpine meadow. Ecology, 93, 2365-2376.

Wilson RM, Hopple AM, Tfaily MM, Sebestyen SD, Schadt CW, Pfeifer-Meister L, Medvedeff C, McFarlane KJ, Kostka JE, Kolton M, Kolka RK, Kluber LA, Keller JK, Guilderson TP, Griffiths NA, Chanton JP, Bridgham SD, Hanson PJ (2016). Stability of peatland carbon to rising temperatures. Nature Communications, 7, 13723. DOI: 10.1038/ncomms13723.

Wu ZT, Dijkstra P, Koch GW, Hungate BA (2012). Biogeochemical and ecological feedbacks in grassland responses to warming. Nature Climate Change, 2, 458-461.

Yu XC, Yao BQ, Zhou HK, Jin YX, Yang YJ, Wang WY, Dong SK, Zhao XQ (2015). Variable responses to long-term simulated warming of underground biomass and carbon allocations of two alpine meadows on the QinghaiTibet Plateau. Chinese Science Bulletin, 60, 379-388. [余 欣超, 姚步青, 周华坤, 金艳霞, 杨月娟, 王文颖, 董世 魁, 赵新全 (2015). 青藏高原两种高寒草甸地下生物量 及其碳分配对长期增温的响应差异. 科学通报, 60 , 379-388.]

Zhu JT (2016). Effects of experimental warming on plant reproductive phenology in Xizang alpine meadow. Chinese Journal of Plant Ecology, 40, 1028-1036. [朱军涛 (2016). 实验增温对藏北高寒草甸植物繁殖物候的影响. 植物生态学报, 40, 1028-1036.]

特邀编委: 温学发 责任编辑: 李 敏 